\title{
Six exercises all senior adults should be doing regularly
}

\section{Conceptual paper}

Aging adults should stay active to live longer Kavanagh ${ }^{1}$ and ward off many of the natural ailments that often accompany the geriatric population. Sarcopenia and chronic diseases can result in diminished mobility, a reduced quality of life, and even premature death. Regular physical activity can help slow the rate of losing muscle mass and strength in aging adults. With regular resistance training, balance and stability are improved, which helps to reduce the chances of falling. ${ }^{2}$ A combination of every type of fitness, including aerobic exercise, muscular strength and endurance training, flexibility exercises, and balance and stability training, is the best recipe for overall physical health. For senior adults, the majority of workouts should include resistance exercises because they are the most effective at preserving the ability to complete basic daily tasks, developing muscle hypertrophy, improving body composition, increasing strength, and enhancing balance and stability.

Resistive training is characterized by short, difficult exercises with resistance that aims to increase muscular strength and endurance. The effects of strength training prevent the development of sarcopenia Evans et al. ${ }^{3}$ and promote hypertrophy in geriatric participants Porter. ${ }^{4}$ Resistance training is beneficial to geriatric individuals because weight lifting can help counter the typical issues that surface as adults age; such as decreased range of motion, reduced strength, and a lack of stability. Resistive training has the ability to improve function, reduce pain, and increase strength in people with osteoarthritis Latham et al. ${ }^{5}$ Women over the age of 80 years old have shown a $40 \%$ reduced chance of falling after engaging in strength and balance exercises CDC. ${ }^{6}$

Limited mobility and a lack of strength and balance in movements associated with basic daily tasks can prevent the completion of essential activities such as going to the grocery store, getting into and out of a car, sweeping, or showering Fleck et al. ${ }^{7}$ The following exercises can help senior individuals increase health, improve balance and body composition, and maintain independence.

The six exercises senior adults should be doing regularly

\section{Aerobic training}

This can be as simple as a walk or a jog, and can be done at the beginning of a workout to warm-up and prepare the body for resistive exercise.

\section{Squats}

With or without weights, a squat may be the most effective way to strengthen legs to get out of a chair, exit a car, and stand up.

\section{Bicep curls}

Having strong biceps helps strengthen arms to complete household tasks like carrying laundry and shopping bags.
Volume II Issue I - 2018

Daniela Benavidez

Baird Middle School, USA

Correspondence: Daniela Benavidez, Baird Middle School, Fresno, Fresno, CA, USA, Tel 559-276-5002, Email TheDoctorBenlsIn@gmail.com

Received: January 28, 2018 | Published: February 09, 2018

\section{Military press}

Possessing the ability to raise items overhead is essential to basic daily tasks such as getting dressed, reaching high shelves, and accessing high cabinet and freezer doors.

\section{Deadlift}

Lifting things up off the ground is a necessary ability for seniors because proper balance and strength can prevent a fall during a basic daily task like pulling up pants or bending over to pick up a laundry basket.

\section{Flexibility training}

Flexibility exercises at the end of a workout that supports range of motion, balance, and stability, can help support a more efficiently moving body, while also helping return the body to homeostasis.

\section{Risks}

While developing and improving flexibility, aerobic capacity, muscular strength, and endurance can be beneficial, there are some risks for injury associated with geriatric weight training. A major concern is progressing without injuring or overusing muscles. ${ }^{7}$ On account of the fact that older adults take longer to recover from resistive training Fleck et al., the strength training programs of seniors require careful progression, as to not increase intensity sooner than the geriatric body can adapt. As with any exercise program, there are also risks of incurring minor injuries. Sprains, strains, and accidental injuries in the trunk and lower body injuries are among the most common injuring among weightlifters Quatman et al. ${ }^{8}$ Using appropriate weight and equipment, proper form, timely progression, variation, and allowing sufficient time for recovery, can all help minimize the instance of injury.

Common ailments among senior individuals include osteoarthritis, diabetes, hypertension, sarcopenia, poor balance, diminished vision, inability to grip, malnourishment, and limited range of motion. These and other special needs can be accommodated to help minimize the risk of injury by adjusting intensity, rest time, exercises, and equipment 
used. Exercise machines, small plastic coated dumbbells, resistive bands, and medicine balls may be appropriate tools for seniors with limitations and in chairs.

\section{Recommendations}

As with all workout regimens, individuals should consult with a primary care physician prior to beginning any new exercise. Depending on specific goals, abilities, and health concerns, geriatric individuals may consider modifying current diet. It is recommended that sedentary female seniors over the age of 50 years old consume 1,600 calories a day, while males in the same age group intake 2,000 kilocalories daily National Institute of Health. ${ }^{9}$ For active seniors engaging in resistive training, an additional $15 \%$ more energy is required to be successful in weight management Campbell et al. ${ }^{10}$ It is important to note that nutritional supplementation that is not supported with resistance training does not improve strength or reduce physical frailty Fiatarone et al., ${ }^{11}$ but may reduce fat and improve body mass index.

\section{Discussion}

The six noted exercises are essential for geriatric health because completing basic daily tasks is the key to retaining independence Benavidez. ${ }^{12}$ Standing up, getting out of a car, lifting, carrying, bending over, and maintaining balance and stability are movements that can be preserved and improved with foundational exercises like squats, bicep curls, military press, dead lifts, and aerobic and flexibility training. With age, the speed of energy consumption at rest, which is known as resting metabolic rate, decreases Fleck et al. ${ }^{7}$ This leaves a positive energy balance in underactive geriatric individuals. As a result, sedentary seniors who maintain caloric intake are at risk for developing obesity and other health complications Campbell et al., ${ }^{2}$ due to a slower functioning metabolism. Being that depression is a common comorbidity in the obese geriatric population Garcia $^{13}$ becoming active can help seniors reduce the risks of developing two preventable diseases due to a slowing body.

\section{Conclusion}

Exercise habits should be consistent and continuous, as the sustained practice of a behavior intended to invoke change is critical to its effectiveness Garcia et al. ${ }^{14}$ Senior adults can participate in six basic exercises to help slow the development of sarcopenia, improve body composition, increase strength and balance, and to diminish the side effects associated with chronic and hypokinetic illnesses like diabetes, hypertension, and osteoarthritis CDC. ${ }^{15}$ While every successful strength training program needs progression, the lack of strength and movement capacity among elderly individuals may hinder the application of the periodization training strategy, but with deteriorating diseases like sarcopenia and osteoarthritis, the intervention of strength training itself is sufficient enough to slow the effects. ${ }^{2}$ The participation in resistive training exercises may be the most influential factor in improving quality of life and health in geriatric adults. This is supported by data that show strength training may also lower the risk of developing type II diabetes, heart disease, hypertension, osteoporosis and obesity. ${ }^{2}$ Combined with a reduced risk of developing osteoporosis, the reduced chances of falling may also prevent fractures and breaks in older, less dense bones. Improving health and increasing strength enables seniors to continue completing basic daily tasks without much assistance, and thus, prolonging independence. Geriatric strength training is healthy, safe, and necessary.

\section{Acknowledgements}

None.

\section{Conflict of interest}

The author declares no conflict of interest.

\section{References}

1. Kavanaugh T. A conditioning program for the elderly. Canadian Family Physician. 1971;17(7):31-33.

2. National Institute of Health. Falls and older adults: Causes and risk factors. USA: National Institute of Health Senior Health; 2013.

3. Evans WJ, Campbell WW. Sarcopenia and age-related changes in body composition and functional capacity. In symposium: Aging and body composition: Technological advances and physiological interrelationships. Journal of Nutrition. 1993;123(2): 465-468.

4. Porter MM. The effects of strength training on sarcopenia. Canadian Journal of Applied Physiology. 2001;26(1):123-141.

5. Latham N, Liu CJ. Strength training in older adults: The benefits for osteoarthritis. Clinical Geriatric Medicine. 2010;26(3):445-459.

6. Centers for Disease Control (CDC). Physical activity. Growing strongerstrength training for older adults: Why strength training? Georgia: Centers for Disease Control; 2011.

7. Fleck SJ, Kraemer WJ. Resistance training for seniors. Designing resistance training programs. 3rd ed. Champaign, USA: Human Kinetics; 2004.

8. Quatman CE, Myer GD, Khoury J, et al. Sex differences in "Weightlifting" injuries presenting to United States emergency rooms. J Strength Cond Res. 2009;23(7):2061-2067.

9. National Institute of Health. Eating well as you get older. USA: National Institute of Health Senior Health; 2014

10. Campbell WW, Crim MC, Young VR, et al. Increased energy requirements and changes in body composition with resistance training in older adults. Am J Clin Nutr. 1994;60(2):167-175.

11. Fiatarone MA, O Neill EF, Ryan ND, et al. Exercise training and nutritional supplementation for physical frailty in very elderly people. $N$ Engl J Med. 1994;330(25):1769-1775.

12. Benavidez D. Improving mood in geriatric individuals. (Doctoral Dissertation). Daphne, Alabama, USA: United States Sports Academy; 2017.

13. Garcia R. Population health management telehealth intervention medical research treating comorbid clinical obesity and depression in geriatric patients part one: Review of tele-medicine scientific research. Research in Medical \& Engineering Sciences. 2017;1(5):1-4.

14. Garcia R, Benavidez D. Transtheoretical model key constructs applied to the intervention \& treatment of weight cycling \& yoyo dieting cognitiveaffective bases of health for weight management. International Journal of Complementary \& Alternative Medicine. 2016;3(3):00071.

15. Centers for Disease Control (CDC). Physical activity. Growing strongerstrength training for older adults: Warm-up. Georgia: Centers for Disease Control; 2011. 